Antimatter and Gravity (WAG 2013)

International Journal of Modern Physics: Conference Series

Vol. 30 (2014) 1460263 (8 pages)

(C) The Author

DOI: $10.1142 / \mathrm{S} 2010194514602634$

\title{
The GBAR experiment
}

\author{
D. P. van der Werf \\ (for the GBAR Collaboration*) \\ Department of Physics, Swansea University, Swansea SA2 8PP, UK \\ D.P.Van.Der.Werf@swansea.ac.uk
}

Received 8 February 2014

Published 7 May 2014

\begin{abstract}
The classical Weak Equivalence Principle has not yet been tested using antimatter in matter gravitational fields. The GBAR (Gravitational Behaviour of Antihydrogen at Rest) experiment, recently approved by CERN, proposes to measure the free-fall acceleration of antihydrogen. In this experiment, positive antihydrogen ions will be produced, and subsequently cooled down using laser cooled $\mathrm{Be}^{+}$ions. Then, when a temperature of around $20 \mu \mathrm{K}$ has been reached, the excess positron will be detached and the free-fall time will be measured using the antiproton annihilation products. An overview of the experiment will be given together with its present status.
\end{abstract}

Keywords: Antimatter; antihydrogen; antiproton; positron; positronium; gravity.

PACS Numbers: 04.90.+e, 07.77.-n, 11.30.Er, 25.43.+t, 36.10.Dr

\section{Introduction}

The classical Weak Equivalence Principle states that the trajectory of a test particle is independent of its composition and internal structure when it is only submitted to gravitational forces. This principle has been tested to a very high precision by many experiments with a great variety of materials and techniques.

This is an Open Access article published by World Scientific Publishing Company. It is distributed under the terms of the Creative Commons Attribution 3.0 (CC-BY) License. Further distribution of this work is permitted, provided the original work is properly cited.

*Department of Physics, Swansea University, Swansea SA2 8PP, UK; CSNSM, CNRS : UMR8609 IN2P3 Université Paris Sud - Paris XI, France;PP, ETHZ, CH-8093 Zürich, Switzerland; Institut Laue-Langevin (ILL), 6 rue Jules Horowitz, F-38042 Grenoble, France; IPCMS, 23 rue du Loess, F-67037 Strasbourg, France; IRFU, CEA, Saclay, F-91191 Gif-sur-Yvette Cedex, France; P. N. Lebedev Physical Institute, 53 Leninsky Prospect, 117924 Moscow, Russia; LKB, CNRS : UMR8552 Université Pierre et Marie Curie - Paris VI Ecole Normale Supérieure de Paris, France; Université d'Evry Val d'Essonne F-91025, France; Atomic Physics Laboratory, RIKEN, 2-1 Hirosawa,Wako, Saitama 351-0198, Japan; Institute of Physics, University of Tokyo, 3-8-1 Komaba, Meguro, Tokyo 153-8902, Japan; Department of Physics, Tokyo University of Science, 1-3 Kagurazaka, Shinjuku, Tokyo 162-8601, Japan. 
Indirect tests of the Equivalence Principle for antimatter have been obtained by comparing the properties of particles and their antiparticles or by arguing about the virtual content of the nuclei of ordinary matter. Two particle-antiparticle systems have been studied in great detail with this aim: comparison of the decay parameters of the $\mathrm{K} 0$ and $\overline{\mathrm{K} 0},{ }^{1}$ and the simultaneous measurement of proton and antiproton cyclotron frequencies. ${ }^{2}$ However, all these tests rely upon disputable theoretical hypothesis - refer for example to a review on experiments and theoretical arguments. ${ }^{3}$

Several experiments have been envisaged in the past to measure the free fall of charged antiparticles (with positrons and antiprotons), but the weakness of gravitational forces makes the protection against electromagnetic influences extremely hard. Measurement with antineutrons can not be made due to the inherent difficulty in slowing them sufficiently down. Mills and Leventhal proposed to test gravity using positronium ${ }^{4}$ in 2002, David Cassidy will describe a roadmap towards such an experiment in this volume.

The simplest form of an antimatter atom is antihydrogen. Before the first production of antihydrogen atoms $(\overline{\mathrm{H}})$ at CERN in $1995^{5}$ and shortly afterwards in Fermilab, ${ }^{6}$ a number of experiments were proposed to measure the gravitational acceleration of the antiatoms $(\overline{\mathrm{g}})$ (e.g. see Refs. 7,8$)$.

The successful production of cold antihydrogen in $2002^{9,10}$ motivated the founding of collaborations with as goal to measure $\bar{g}$. The subsequent trapping experiments on the anti-atom, ${ }^{11-13}$ lead to the first direct measurement of $\bar{g}^{14}$ which as result that the absolute value of the ratio between the gravitational and inertial mass of the anti-atom is smaller then 75 .

In 2007 a letter of intend was submitted to the SPSC committee, ${ }^{15}$ proposing a measurement of $\bar{g}$ using $\overline{\mathrm{H}}^{+}$after an idea from Walz and Hänsch. ${ }^{16}$ This letter was followed by a detailed proposal for an experiment to measure $\bar{g}$ by the GBAR collaboration in 2011. ${ }^{17}$ The approval by CERN came in 2012, and GBAR is scheduled to start measuring in 2017-2018. In the following we will describe the GBAR experiment. The proposed experiments by AEGIS and ALPHA will described in separate articles in this volume.

\section{Measurement Principle}

Instead of using a neutral atom, GBAR plans to use an $\overline{\mathrm{H}}^{+}$ion, where charged particles are relatively easily to manipulate and to cool down to low temperatures. The anti-ion will be produced via the reactions:

$$
\begin{aligned}
& \overline{\mathrm{p}}+\mathrm{Ps} \rightarrow \overline{\mathrm{H}}+\mathrm{e}^{-} \\
& \overline{\mathrm{H}}+\mathrm{Ps} \rightarrow \overline{\mathrm{H}}^{+}+\mathrm{e}^{-},
\end{aligned}
$$

and will subsequently be trapped and sympathetically cooled in a Paul trap, whereafter the ion is neutralised using laser radiation. The neutral anti-atom will then "fall", and after some time, reach a surface where the antiproton annihilates with 
a proton or neutron producing pions. The charged pions will be observed using a position sensitive detector. The time between positron removal and pion detection will give a measure of $\bar{g}$.

\section{Experimental Details}

An schematic overview of the experiment is shown in Fig. 1(top), together with the layout of the experimental equipment within the AD hall (bottom). Electrons, accelerated to about $20 \mathrm{MeV}$ in the Linac, hit a beam target and generate fast positrons via pair production. A moderator in the form of either a tungsten foil

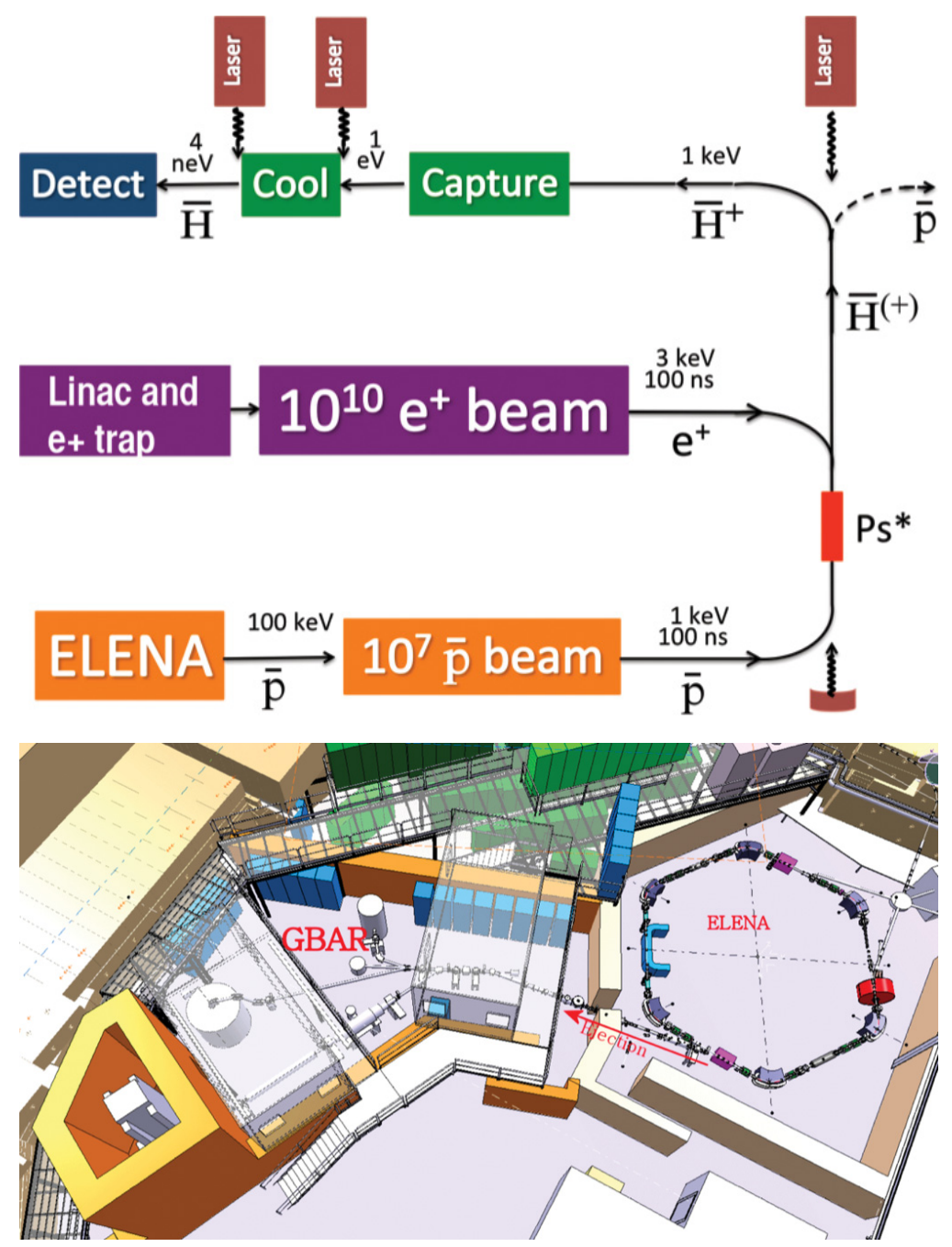

Fig. 1. (Color online) Schematic overview of the GBAR experiment (top) and layout of the equipment within the $\mathrm{AD}$ hall (bottom). 


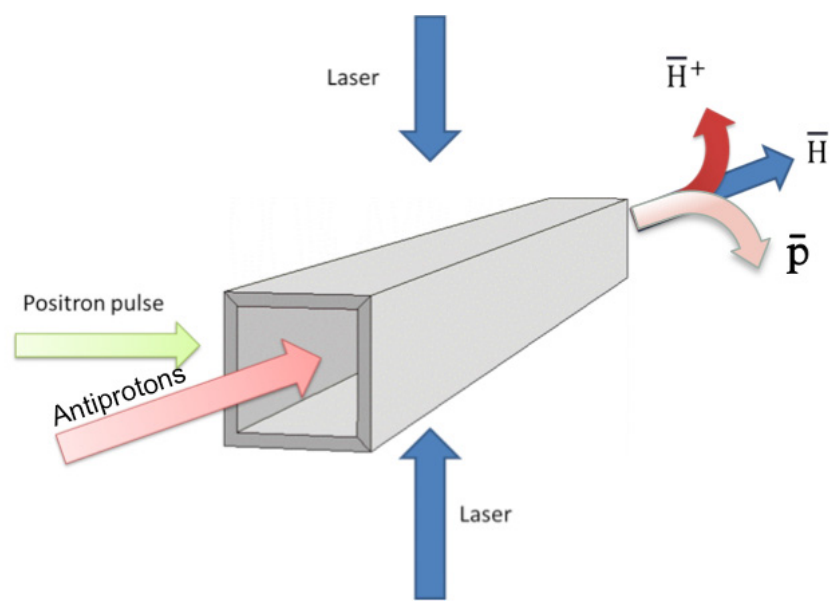

Fig. 2. (Color online) Schematic diagram of the interaction chamber. The surface of the chamber is coated with porous silica, used for producing positronium.

or mesh, or a rare gas moderator slows the positrons down whereafter they are trapped in a Penning type trap. A preloaded electron plasma cools the positrons down quickly enough so the next bunch of positrons can enter without loosing the already trapped ones. This process is repeated until there are about $10^{10}$ positrons accumulated. Then, when needed, the positrons are ejected from the trap, accelerated and bunched towards the reaction chamber. In the reaction chamber wall, consisting of mesoporous silica, positronium will be formed and escape into the vacuum in a form of a cloud.

Before positronium formation, a bunch containing about ten million, $100 \mathrm{keV}$ antiprotons originating from ELENA will be slowed down to the order of keV using a drift tube. The antiparticles will then arrive in the reaction chamber just as the Ps cloud is emitted from the porous surface(see Fig. 2), and some of the antiprotons will react via equations (1) and (2) to form $\overline{\mathrm{H}}^{+}$. The reaction chamber width will be of the order of $1 \mathrm{~mm}$ so careful control of the beam parameters coming from Elena are essential.

Comini and Hervieux ${ }^{18}$ recently showed that the $\overline{\mathrm{H}}^{+}$formation cross section depends on the impact energy of the antiproton and the quantum state of the positronium. For instance, one photon excitation (see Fig. 3a) of about $20 \%$ of the positronium atoms to the $2 \mathrm{p}$ state, combined with a antiproton impact energy to about $2 \mathrm{keV}$, could improve the $\overline{\mathrm{H}}^{+}$production with a factor of 5 compared to using ground state positronium (see Fig. 3b). In the latter case the minimum impact energy of the antiproton needs to be about $6 \mathrm{keV}$ to fulfil the energy and momentum conservation laws. Similarly, excitation of $40 \%$ of the Ps to a 3 d state increases the formation cross section as well, with the added advantage that the lifetime of the positronium in the $3 \mathrm{~d}$ state (not the annihilation lifetime) is 31 ns while that one of the 2 p state is only $3.2 \mathrm{~ns}$. So, in the first case a $410 \mathrm{~nm}$ laser pulse of $50 \mathrm{~ns}$ 

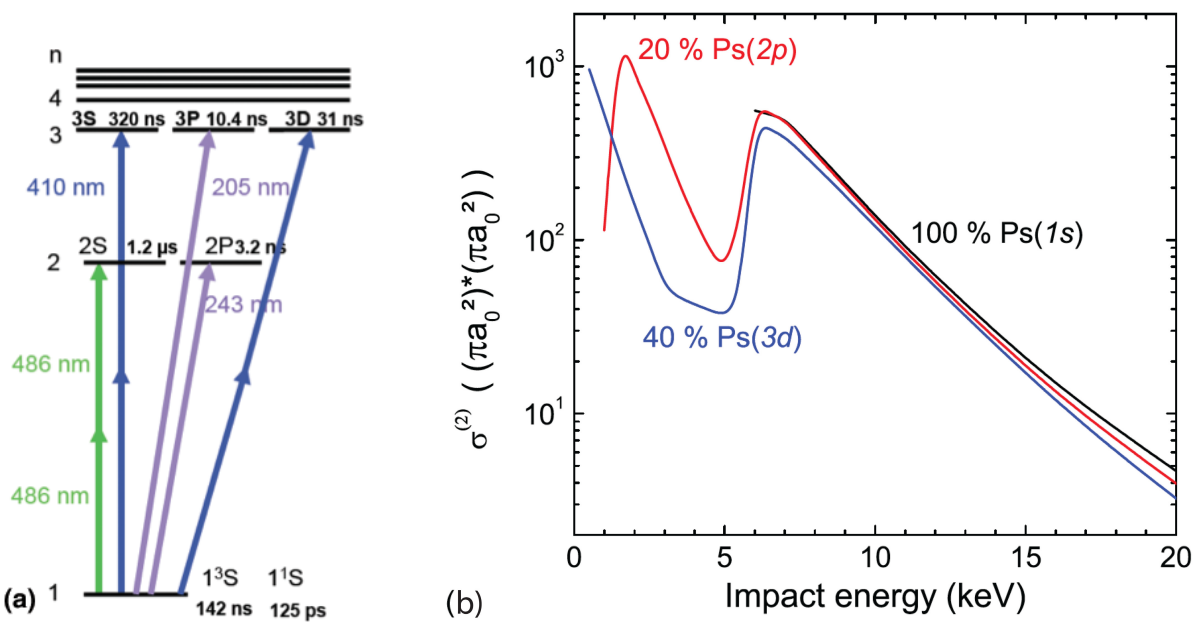

Fig. 3. (Color online) Effect of excited positronium states on the total $\overline{\mathrm{H}}^{+}$formation cross section. a) Possible positronium excitation routes. b) Total $\overline{\mathrm{H}}^{+}$formation cross sections for ground state positronium (black), $20 \%$ of the positronium excited to $2 \mathrm{p}$ (red) and $40 \%$ excited to $3 \mathrm{~d}$ (blue). ${ }^{18}$

duration will suffice while for the $2 \mathrm{p}$ excitation $243 \mathrm{~nm}$ pulses, repeated every couple of nanoseconds is needed.

Most of the antiprotons passing through the positronium cloud will not undergo a reaction with positronium and will pas unhindered through the chamber. A fair amount of the antiprotons will form neutral antihydrogen and exit the chamber before undergoing a second reaction. Only a small number of $\overline{\mathrm{H}}^{+}$will be formed in each pulse of antiprotons. Because of the different charges, the $\overline{\mathrm{p}}, \overline{\mathrm{H}}$ and $\overline{\mathrm{H}}^{+}$ will spatially be separated using a bending magnet. There could well be uses for the antiproton and antihydrogen beams, and provisions and space will be made to expedite possible future experiments.

After separation, the positive $\overline{\mathrm{H}}$ ions will be trapped in Paul trap and then cooled from the order of keV to energy of $3 \mathrm{neV}$, in two stages. The first stage consists of a capture trap, preloaded with around $10,000 \mathrm{Be}^{+}$ions which are laser cooled with $313 \mathrm{~nm}$ laser light. The $\overline{\mathrm{H}}^{+}$will be sympathetically cooled by the $\mathrm{Be}^{+}$ions until a energy of a few meV has been reached. Subsequently, one $\mathrm{Be}^{+} / \overline{\mathrm{H}}^{+}$pair will be transferred into a precision trap were further cooling will take place using Raman side band cooling. A more detailed description of the Paul traps and cooling lasers to be used is given in the article from Hilico in this volume.

Once a equivalent temperature of about $20 \mu \mathrm{K}$, corresponding with a speed of about $1 \mathrm{~ms}^{-1}$, has been reached, one of the positrons of the $\overline{\mathrm{H}}^{+}$ion is detached by means of a laser pulse, leaving a neutral $\overline{\mathrm{H}}$ atom which then start to move due to the gravitational force. The moment of photo detachment is simultaneously the start time of the measurement, the stop time is determined by the detection of one or more of the charged pions originating from the annihilation of the antiproton with 


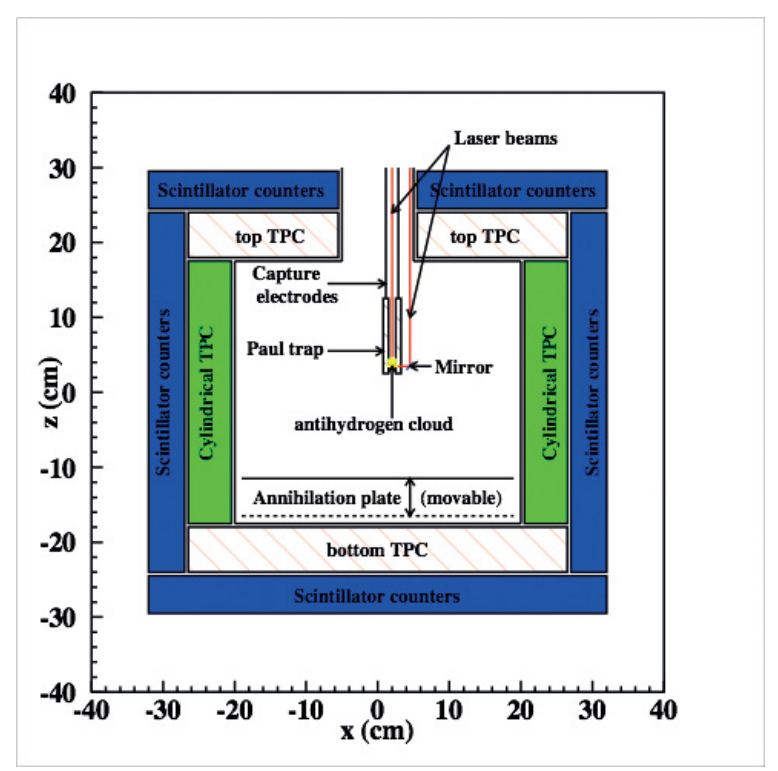

Fig. 4. (Color online) Schematic diagram of the free fall chamber.

a proton or neutron (see Fig. 4). These pions will be detected using time projection chambers surrounding the vacuum vessel in which the precision trap is housed.

The binding energy of $\overline{\mathrm{H}}^{+}$is $0.76 \mathrm{eV}$, which corresponds with a wavelength of $1.64 \mu \mathrm{m}$. The detaching process gives recoil of about $0.3 \mathrm{~ms}^{-1}$ to the neutral antiatom, adding a couple of centimetres in the horizontal plane after a free fall of 15 $\mathrm{cm}$. Simulations (see Fig. 5) have shown ${ }^{17}$ that a statistic accuracy for $\Delta \overline{\mathrm{g}} / \overline{\mathrm{g}} \sim 10^{-2}$ can be reached with about 1500 free-falling $\overline{\mathrm{H}}$ atoms.

In the longer term, after the initial gravity measurements, the accuracy will be improved using gravitational quantum levels of the antihydrogen atom. At very low energies the antihydrogen atoms will, for the right surface, undergo quantum reflection. This procces depends strongly on the energy, for instance, an antihydrogen atom with a kinetic energy equivalent to a potential gravitational energy of about $100 \mu \mathrm{m}$ (if $\overline{\mathrm{g}}=\mathrm{g}$ ) will be reflected form a silica surface $96 \%$ of the time. For these, an energy shaping device, ${ }^{19}$ like shown in Fig. 6 , has been envisaged to select only $\overline{\mathrm{H}}$ with an vertical velocity low enough to bounce between the two discs. Simulations have shown that in this case the accuray for $\Delta \overline{\mathrm{g}} / \overline{\mathrm{g}}$ could reach levels down to $10^{-3}$. More information is given in the contributions from Dufour and Voronin in this workshop.

\section{Status}

At Saclay, a $5 \mathrm{MeV}$ demonstration Linac, including the positron production and moderation stages have been build. The positron trap, called the multi ring trap 


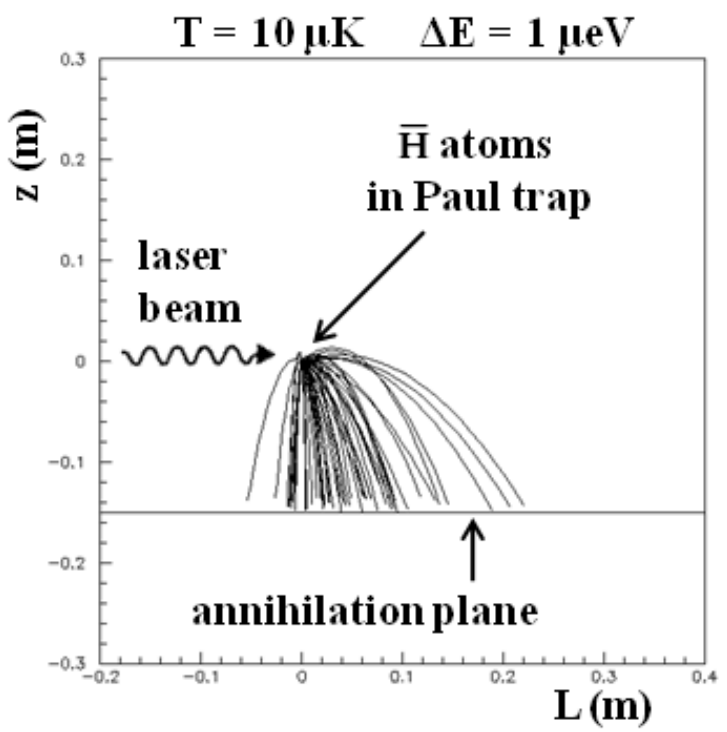

Fig. 5. Trajectories for antihydrogen atoms after the excess positron has been removed by a laser pulse (assuming $\bar{g}=\mathrm{g}$ ).

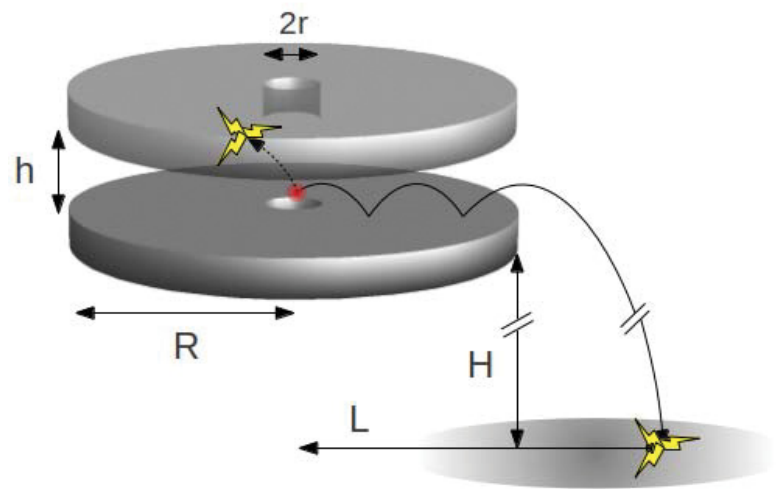

Fig. 6. (Color online) Possible shaping device able to select very low energy antihydrogen atoms using quantum reflection. ${ }^{19}$

and was used before at RIKEN for similar purposes,${ }^{20}$ has been installed. Positrons have been produced and experiments including positron trapping and stacking and positronium formation are under way. A $410 \mathrm{~nm}$ pulsed laser system is under construction at Laboratoire Kastler Brossel and will soon be installed at Saclay which will be used to excite the potitronium atoms. 
Because ELENA will not deliver antiprotons for experiments until 2017, formation cross sections measurement for $\mathrm{H}$ and $\mathrm{H}^{-}$will be performed at Saclay, the results of which will be used to inform the final design of the reaction chamber and its accompanying lasers.

The Paul traps, including the necessary lasers, are under design and the trapping and cooling process will be tested using $\mathrm{H}^{+}$and $\mathrm{H}_{2}^{+}$ions.

GBAR will start installation and commissioning of the equipment at CERN in 2015. Hopefully the first physics experiments will take place in 2017 when ELENA will come online.

\section{Acknowledgments}

The author would like to thank the Leverhulme Trust for the Research Fellowship award RF-2012-495, and the EPSRC for its overall antihydrogen research support, currently via award EP/H026932/1.

\section{References}

1. A. Apostolakis et al., Phys. Lett. B 452, 425 (1999).

2. G. Gabrielse, A. Khabbaz, D. S. Hall, C. Heimann, H. Kalinowsky and W. Jhe, Phys. Rev. Lett. 82, 3198 (1999).

3. M. M. Nieto and T. Goldman, Physics Reports 205, 221 (1991).

4. A. P. Mills Jr. and M. Leventhal, Nucl. Instr. Meth. Phys. Res. B 192, 102 (2002).

5. G. Baur et al., Phys. Lett. B 368, 251 (1996).

6. G. Blanford, D. C. Christian, K. Gollwitzer, M. Mandelkern, C. T. Munger, J. Schultz and G. Zioulas, Phys. Rev. Lett. 80, 3037 (1998).

7. R. Poggiani, Hyperfine Interact. 76, 371 (1993).

8. T. J. Phillips, Hyperfine Interact. 100, 163 (1996).

9. M. Amoretti et al., Nature 419, 456 (2002).

10. G. Gabrielse et al., Phys. Rev. Lett. 89 (2002).

11. G. B. Andresen et al., Nature 468, 673 (2010).

12. G. B. Andresen et al., Nature Physics 7, 558 (2011).

13. C. Amole et al., Nature 483, 439 (2012).

14. C. Amole et al., Nature Communications 4, 1785 (2013).

15. P. Pérez et al., Tech. Report SPSC-I-237. CERN-SPSC-2007-038 (Nov 2007), revised version submitted on 2007-11-30 17:05:11.

16. J. Walz and T. Hänsch, General Relativity and Gravitation 36, 561 (2004).

17. G. Chardin et al., Tech. Report CERN-SPSC-2011-029. SPSC-P-342, CERN (Geneva, 2011).

18. P. Comini and P. A. Hervieux, New J. Phys. 15, 095022 (2013).

19. G. Dufour, P. Debu, A. Lambrecht, V. V. Nesvizhevsky, S. Reynaud and A. Y. Voronin, Eur. Phys. J. C 74, 2731 (2014).

20. N. Oshima et al., Phys. Rev. Lett. 93, 195001 (2004). 